\title{
Paulownien - attraktive Nutzhölzer und Wappenbäume
}

\author{
Veit Dörken \& Hilke Steinecke
}

\begin{abstract}
Paulownia tomentosa (Princess Tree) is grown in parks and gardens. Its biology, use as ornamental and timber tree and its symboly are outlined.
\end{abstract}

\section{Zusammenfassung}

Paulownia tomentosa (Blauglockenbaum) ist ein beliebter Parkbaum. Biologie, Verwendung als Zierbaum, Nutzung des Holzes und Symbolik werden erläutert.

\section{Baum mit Riesenblättern}

Jährlich im Herbst wird man als Botaniker gefragt, welche außergewöhnlichen Pflanzen so extrem große herzförmige Blätter bilden und einen Jahreszuwachs von mehreren Metern aufweisen. Manchmal landen auch Briefumschläge, gefüllt mit liebevoll gefalteten Laubblättern, auf dem Schreibtisch. Die Absender wollen dann außer dem Artnamen gelegentlich auch wissen, ob ein tropisches Gewächs oder gar eine gentechnisch veränderte Pflanze vorliegt. Dabei handelt es sich um Blätter des Kaiser-Blauglockenbaumes (Paulownia tomentosa), der schon seit Jahren bei uns als smogverträglicher Straßen- und Parkbaum gepflanzt wird (Schmidt 1999). In Frankfurt stehen schöne und auffälli-

Abb. 1: Paulownia fargesii im Palmengarten, Frühjahr 2013.

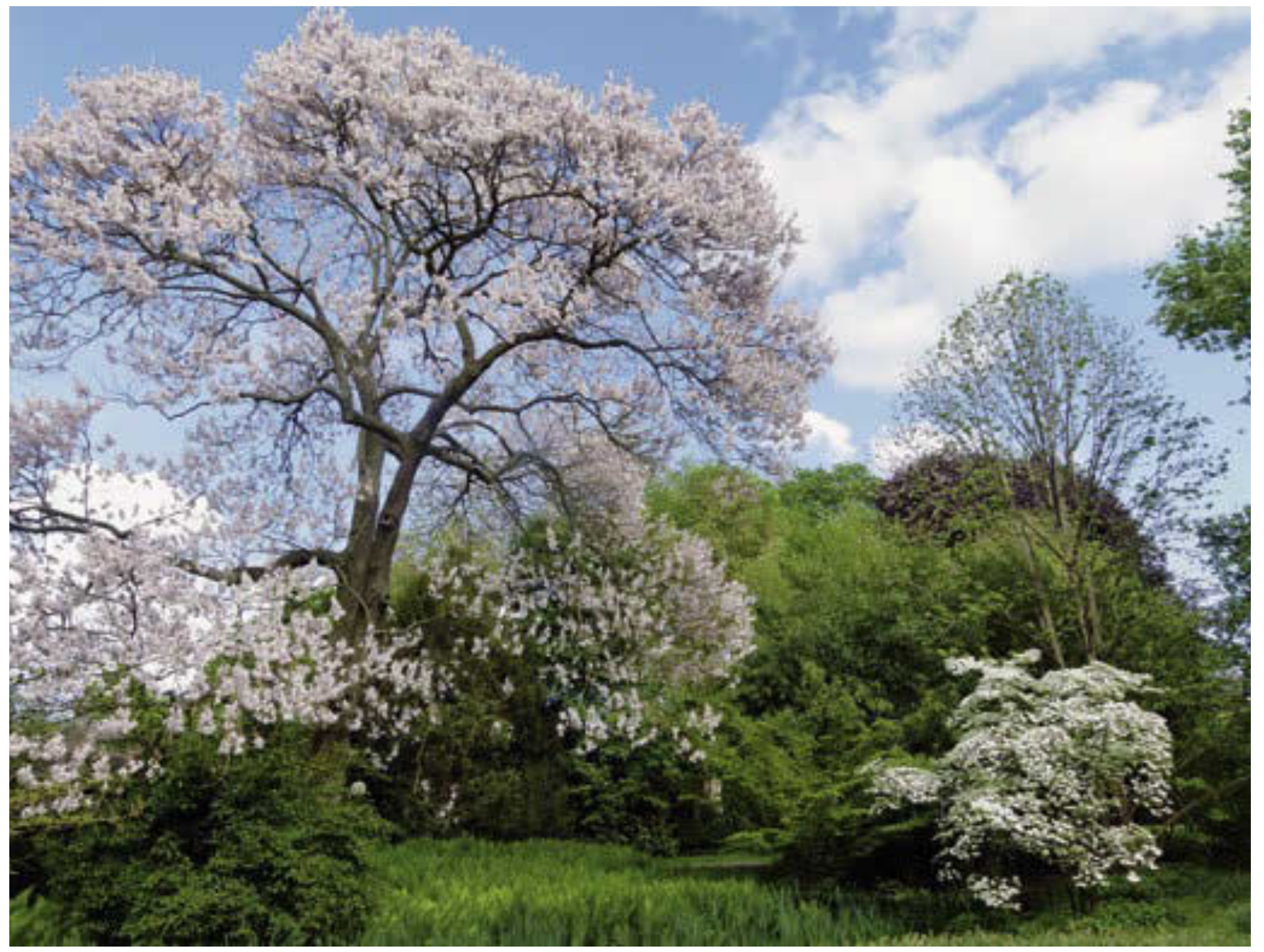



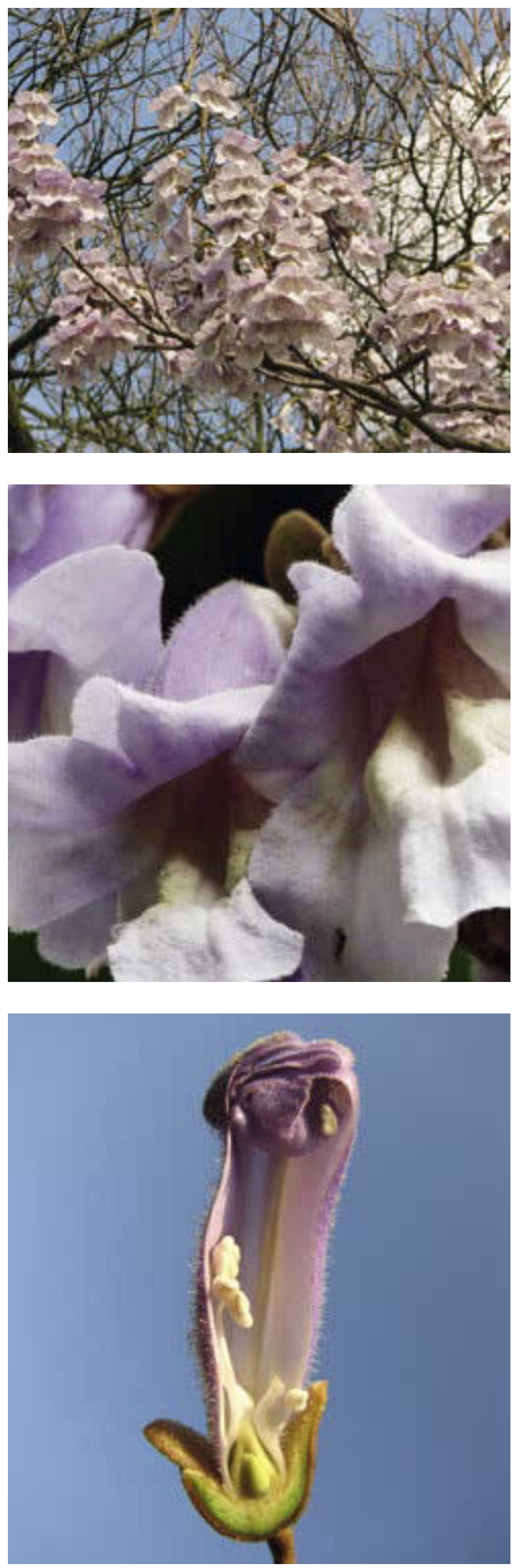

ge Paulownien auch in den Anlagen am Mainufer, u.a. im wärmebegünstigten Nizza. Besonders in Städten mit einem milden Lokalklima wie z. B. Bonn oder Frankfurt samt er sich teilweise extrem stark aus, Jungpflanzen laufen auf Ruderalflächen auf oder zwängen sich aus Mauerritzen dem Licht entgegen. Schösslinge und Wurzelbrut erreichen dabei einen erstaunlichen Jahreszuwachs von $2 \mathrm{~m}$ und mehr, ihre Blätter werden über $40 \mathrm{~cm}$ breit. Die großen Blätter erlauben eine effektive Photosynthese, weshalb die großen Jahreszuwächse möglich sind. Diese rasch wüchsigen Sämlinge können allerdings bei zu früh einsetzenden Herbstfrösten stark geschädigt werden.

\section{Ein Baum der Kaiser und Prinzessin}

Die Gattung Paulownia umfasst 7 in Ost-Asien heimische Baum-Arten. Verbreitungsschwerpunkt ist China, wobei das Gattungsareal bis nach Taiwan, Nord-Vietnam und Laos reicht. Im Jahr 1834 wurde Paulownia im Botanischen Garten Paris eingeführt (BEAN 1950). Lange wurde die Gattung zu den Rachenblütlern (Scrophulariaceae) gestellt, bildete mit ihrer Lebensform Baum jedoch innerhalb der Familie eine Ausnahme. Aktuell gilt Paulownia als einzige Gattung innerhalb der eigenen Familie Paulowniaceae. Der Gattungsname Paulownia geht auf den Botaniker, Pflanzenjäger und Japanforscher Philipp Franz von Siebold zurück, der den Kaiser-Blauglockenbaum (Paulownia tomentosa $=P$. imperialis) 1835 nach der russischen Prinzessin und späteren Großfürstin Anna Paulowna von Holstein-GottorpRomanow (1795-1865) benannt hat. Siebold stand in Diensten ihres Vaters Zar PAUL I. (1754-1801). Auf diesen adeligen Namen und vermutlich auch auf die Schönheit der Blüten beziehen sich die Bezeichnungen Empress Tree (Kaiserbaum) und Princess Tree (Prinzessinnenbaum). Auch Franz Joseph I. (1830-1916),

Abb. 2 (oben): Blühende Paulownia tomentosa.

Abb. 3 (Mitte): Blüten von Paulownia tomentosa.

Abb. 4 (unten): Geöffnete Blüte vom

Paulownia tomentosa. 
österreichisch-ungarischer Kaiser, war von der Schönheit des Baumes sehr angetan und ließ ihn an verschiedenen Stellen rund um Wien pflanzen, so auch in Schönbrunn.

Der Blauglockenbaum wird auch schon lange in Japan als kaiserlicher Baum verehrt. Die stilisierten Blüten und Blätter sind im kaiserlichen Wappen (Go-Shichi No Kiri) sowie im Wappen des japanischen Premierministers enthalten. Seit 1888 verleiht die japanische Regierung den Orden der Paulownienblüte für hervorragende militärische oder zivile Verdienste. Zusammen mit dem Orden der aufgehenden Sonne und dem Chrysanthemenorden gehört er zu den drei wichtigsten japanischen Auszeichnungen. Der Blauglockenbaum ist Symbolpflanze der Universität Tsukuba und ziert ihr Logo. Auch auf der japanischen 500-YenMünze ist er abgebildet.

\section{Paulownia tomentosa}

Blauglockenbäume erfreuen je nach Wuchsort und Witterung von April bis Mai noch vor dem Laubaustrieb mit ihrem auffälligen üppigen Blütenflor. Im Palmengarten fiel im Frühjahr 2013 Paulownia fargesiii durch eine diesmal besonders üppige Blütenpracht auf. Wegen der kühlen Witterung war der am Bachlauf wachsende Baum über 2 Wochen ein besonderer Blickfang. Paulownia fargesii ist $P$. tomentosa sehr ähnlich, allerdings sind die Blüten blasslavendelblau bis weiß gefärbt.

Paulownia tomentosa wird $12(-15) \mathrm{m}$ hoch und ist wenig verzweigt. Ihre jungen Triebe sind meist stark behaart. Die großen, breiteiförmigen Blätter sind $30(-35) \mathrm{cm}$ lang und fast genauso breit. Sie sind entweder ganzrandig oder schwach 3- (bis 5-)lappig. In ihrer Form ähneln sie etwas den Blättern von Trompetenbäumen (Catalpa). Sowohl die Blattober- als auch die -unterseite sind behaart. Die Blüten sind hell- oder lavendelblau, duftend und glockenförmig (dt. Name!). Die Rachenblumen ähneln in ihrer Form den Blüten des Fingerhuts (Digitalis), werden 4 bis $5 \mathrm{~cm}$ lang und stehen in aufrechten $30(-40) \mathrm{cm}$ langen Rispen. Die Stiele der Blütenstände sind wie auch die kugeligen Blütenknospen dicht gelbfilzig behaart
(KrüSSMANN 1978). Außer in der Hauptblütezeit im Frühling können in einem milden Herbst zusätzlich einzelne Blüten, ähnlich wie bei Rosskastanien, erscheinen. Die ersten Blüten sind bereits im Alter von 8 bis 10 Jahren zu erwarten. Die Kapselfrüchte öffnen sich 2-klappig und enthalten über 1000 kleine geflügelte Samen, die wegen des geringen Eigengewichtes durch Wind weit von der Mutterpflanze entfernt ausgebreitet werden. Die Samen enthalten reichlich Öl, das vor allem in Japan für die Herstellung von hochwertigen, schnell trocknenden Lacken beliebt ist (BäRNER 1962). Der sehr widerstandsfähige Kaiser-Blauglockenbaum wird wegen seiner schnellen Ausbreitung mancherorts als aggressiver Neophyt betrachtet. Er verwildert in Europa sowie in Nordamerika. In der Schweiz kommt er vor allem im wärmebegünstigten Süden vor. Neuerdings gibt es aber die Hybride 'Nordmax 21', die sich nicht mehr unkontrolliert ausbreiten soll und vielleicht demnächst häufiger in Baumschulen anzutreffen ist. Denn beliebt sind Paulownien nach wie vor: Paulownia tomentosa ist aufgrund der massenhaft hervorgebrachten Blüten, des weit wahrnehmbaren Duftes, der großen Blätter sowie der lang haftenden Früchte ein spektakuläres, exotisch anmutendes Solitärgehölz. Bei kräftigen Spätfrösten kann die Blüte durch Erfrieren der Knospen allerdings ausfallen. Da zunächst nur wenig in Festigungsgewebe investiert wird, sind die jungen Sprossachsen entsprechend unausgereift den Frühfrosten ausgesetzt. Nicht selten frieren junge Paulownien stark zurück.

\section{Holz}

Der Blauglockenbaum gehört zu den weltweit am schnellsten wachsenden Wertholzbäumen. Zunehmend wird vor allem in Asien überlegt, ihn zur Deckung des Holzbedarfs in Plantagen anzubauen. Das Holz wird, wie auch der Baum in Japan, als Kiri bezeichnet (MabberLey 2008). Es trocknet schnell, ohne sich dabei zu verziehen. Es ist hart, stabil, dabei sehr leicht bei einer Dichte von $0,25-0,3 \mathrm{~g} / \mathrm{cm}^{3}$. Zudem ist das Holz harzfrei und schwer entflammbar (BÄrner 1962). Es eignet sich zum Anfertigen 

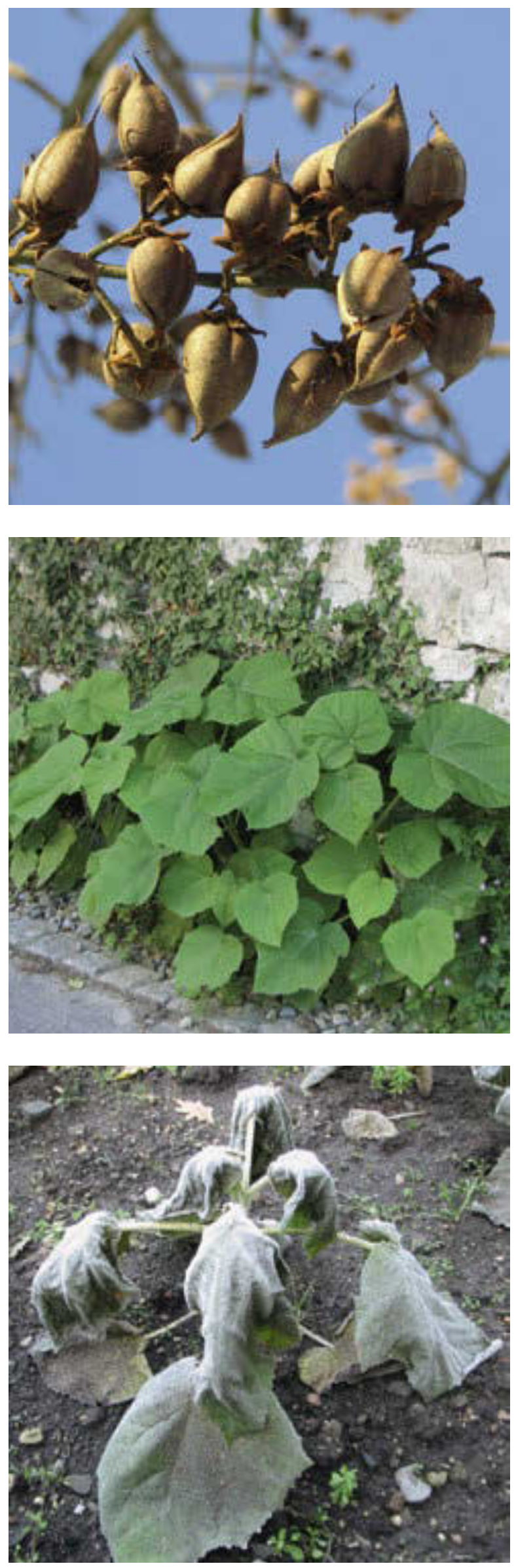

von Möbeln, Musikinstrumenten, Booten, Flugzeuginventar, Holzspielzeug, Tischtennisplatten, oder in Japan von feuersichereren Schränken für wertvolle Kimonos. In Japan werden auch Esstische, Tassen- und Teebretter aus Kiri hergestellt. In China werden Blauglockenbäume schon seit Langem traditionell hoch geschätzt. So galten vor einem Haus gepflanzte, blühende Paulownien für Reisende als ein Hinweis auf ein heiratsfähiges Mädchen. Denn zur Geburt eines Mädchens wurde ein Blauglockenbaum gepflanzt, der sich zu einem stattlichen Baum entwickelt hatte, wenn das Mädchen ins heiratsfähige Alter kam. Zur Hochzeit wurde der Baum gefällt, um aus dessen Holz zahllose Gegenstände für die Mitgift herzustellen. Auch die schwarzen Lackschuhe, die buddhistische Shinto-Priester in Japan bei rituellen Handlungen tragen, sind aus Paulownien-Holz gefertigt. Deren Glaube untersagt das Töten von Tieren und Lederschuhe dürfen folglich in Tempeln nicht getragen werden, sodass auf Holz ausgewichen werden muss.

\section{Literatur}

BÄRNER, J. 1962: Die Nutzhölzer der Welt. - Weinheim. BEAN, W. J. 1950: Trees and shrubs hardy in the British Isles. - London.

KRÜssManN, G. 1978: Handbuch der Laubgehölze. Berlin, Hamburg.

Mabberley, D. J. 2008: Mabberley's plant book. Cambridge.

Schmidt, W. 1999: Gehölze für mediterrane Gärten. Stuttgart.

\section{Internetseiten}

Blauglockenbäume - Chinesischer Blauglockenbaum http://www.garten-treffpunkt.de/lexikon/ blauglockenbaum.aspx

http://www.theplantlist.org/

Abb. 5 (oben): Früchte von Paulownia tomentosa.

Abb. 6 (Mitte): Schösslinge von Paulownia tomentosa.

Abb. 7 (unten): Nach dem ersten Frost erfrorene Schösslinge von Paulownia tomentosa. 